\title{
Review of: "The Impact of Financial Development On The Clean Energy Transition In MENA Region: The Role of Institutional And Political Factors."
}

yan tan

Potential competing interests: The author(s) declared that no potential competing interests exist.

1. The introduction is well written and puts their paper in context. However, the 10th paragraph states: "More specifically, the MENA region is rich in fossil fuels and is considered the world's largest supplier of oil and gas (Saidi et al.2020). For oil importing-countries in this region, reducing oil imports is an important issue to cope with threats associated with price volatility, as the instability of oil prices negatively affects their economic performance. Therefore, the transition to clean energy will reduce the oil dependence of non-exporting countries (Atalay et al., 2016)." I don't quite understand if the MENA is rich in fossil fuels and the world's biggest supplier of oil and gas, it means that they are oil exporting countries in the region. Is there any contradictions in this sentence? Moreover, please reorganize the paragraphs or combine some paragraphs together since there are only one or two sentences in one paragraph only.

2.About the literature review, this subsection is adequate but disordered. First, the authors listed four hypothesis of the relationship between renewable energy and economic growth, which I don's see any connections with the title. What is the purpose of the author to discuss renewable energy and economic growth here when your title is about financial development, institutional and political factors. Second, for the rest of this part, the author just simply stated the main ideas of the previous literature, but I expect more discussions and evaluations about the previous literature. The contribution of the paper should also be built on the assessment of the research gap of the previous papers. I do not see enough discussions here in this part.

3. Methodology and data section. First, In the first paragraph, the author stated that "this study points to a general index of financial development. This index includes nine indicators that summarize the level of development of financial institutions and markets in terms of depth, access and efficiency." However, I don't see the nine indicators listed one by one. If the author claims that this special indicator is the contribution of the paper, please make it clear and explain it clearly in part why it is different from or more efficient than the existing indicators. Second, regarding the institutional quality, "This variable includes corruption, law and order, bureaucratic quality, democratic accountability and stability of government..... Indeed, the aggregate use of the different measures gives a more accurate effect than an isolated variable." I don't think so, since it might cause aggregated bias. And how do the author combine it? Or the WDI provide a general indicator? Please explain it. Third, please give detailed model specifications.

4. In the empirical results section, the pre-estimation tests and the results are complete. 
5. Finally, the conclusion section and abstract are complete.

In conclusion, I don't think this paper is with adequate academic contribution to publish in the journal. The main reasons are as follows:

First, the logic between each each part is weak, there is no enough state quo discussion either, which can hardly convince the reader why the case of MENA region is so interesting and urgent.

Second, there is no enough assessment and discussion of the existing literature, and there is a lack of the comparison between the existing literature and current paper.

Third, please format and edit the paper CAREFULLY before submission as the basic academic standard. Fourth, there is no interesting innovations in selecting the variables. 